in advance: the greatest concentration and discrimination will be exercised by the contributors : dissertations will be rejected as having no proper place in periodicals. It is expected in consequence "that periodicals inflated in size and price, especially in the field of medicine and natural sciences, will be cut down from 1934 onwards by at least $20 \%$ in size and price as compared with the position in 1933". The Börsenverein declares that it will regard non-observance of this agreement as "an offence against the statutory duties of its members".

\section{Thomas Young and Colour Mixtures}

For the Thomas Young Oration delivered before the Physical Society on October 6, Dr. Herbert Ives chose for his subject "Thomas Young and the Simplification of the Artist's Palette". The experimentally well established fact enunciated by Thomas Young that all colours may be matched by the mixture of three properly selected primaries, has been extensively used in colour photography and typographic printing. It has not, however, been heretofore successfully used in painting. The simplification indicated by the three-colour principle has been retarded in realisation largely owing to the mistaken, but widely held, belief that the primary pigment colours are red, yellow and blue. Actually the pigment primaries, which act by subtraction or absorption of light from white, should be complementary in hue to the red, green and blue, which are the primaries for mixing light by addition. Those colours are minus red (spectrum minus red) or turquoise, a minus green or crimson, a minus blue or yellow, each having wide overlapping spectral reflection bands. Pigments of these colours, of proper spectral characteristics, are capable of mixing in pairs to make red, green and blue, and all three together to make black. When mixed with white all variations of saturation and hue are obtained. The practical problem consists in procuring pigments possessing the indicated spectral reflectivities, and having satisfactory chemical properties, such as freedom from reaction with the oil or other medium, and satisfactory permanence. Due to the very great advances which have been made in the dye industry to meet recent demands for permanent colours for automobiles and outdoor signs, it is now possible to select pigments nearly enough meeting the scientific requirements to test the practicability of the principle. This has been done with success, and pictures so painted were exhibited in connexion at the meeting. The great advantage of a three-colour palette is its simplicity and freedom from ambiguity.

\section{Diet and Cancer}

MaNY food products of the most varied kinds have been incriminated from time to time as being causative of the supposed increase of cancer. One of these in particular is the tomato, and Bellows and Askanazy of Geneva have stated that they have produced in white rats cancer of the sarcoma type by injection of tomato juice or of a particular bacillus obtained from the tomato, and also by feeding the animals with the juice. In a recent communication in the Lancet (Sept. 23, p. 698) M. J. A. des Ligneris records attempts to confirm these observations in South Africa, with completely negative results. Others have also similarly failed, and it is mentioned that Askanazy himself in another series of experiments obtained no further positive results. It is suggested that the presence or absence of some constitutional factor in the experimental animals determines whether or no cancer develops. If this factor is present, any adequate chronic irritation, such as that produced by tomato juice, suffices to induce cancer formation.

\section{The Bureau of Mines and the U.S. Petroleum Industry}

Mr. H. C. Fowlen in his paper on "Petroleum and Natural Gas Studies of the United States Bureau of Mines" (Information Circular 6737, July 1933) gives an important résumé of the work of the petroleum and natural gas division of the U.S. Bureau of Mines. The chief motive of the Bureau is to collect and correlate the best available knowledge and information regarding physical phenomena, and thereafter make analyses of the data received with the view of delineating laws and fundamental relationships having practical application to problems of economic production, transport. ation and manufacture of gas and oil.. During recent years, with the rapid growth of the industry, innumerable complex problems have arisen and in consequence the scope of the Bureau's work has changed and enlarged materially since its inception in 1914. The technique of practical oil and gas production is now more generally known and the Bureau is, therefore, able to concentrate on studies of a fundamental nature and on problems incapable of solution by individual concerns. Technical problems now confronting the Bureau concern refining ; production of gas and oil, including related problems of pipe-line transportation of natural gas ; engineering field studies; and special engineering problems, including technical research for information sought by the industry. The chief value of the Bureau's work, however, lies not in the multiplicity of problems under investigation but in the fact that all published information is definitely based on results of commercially independent experimentation and investigation. No statistics or data are published without being first subjected to rigorous scrutiny within the Bureau, and frequently such information is critically reviewed by recognised national authorities in the industry before release. Finally, the Bureau places its detailed and valuable findings without reservation at the disposal of a world-wide technical public, long since mindful of its obligation to a most efficient and learned body.

\section{Suspension of Excavation at Verulamium}

AT the close of the present season, the excavations on the prehistoric and Roman sites of Verulamium at St. Albans, it is announced, will be suspended for a period of indefinite duration. The Excavations Committee, under the supervision of which the work 